\title{
EARLY VALIDATION OF REQUIREMENTS
}

\author{
A Case Study Using Formal Methods
}

\author{
Steven P. Miller \\ Rockwell Collins, Inc. \\ spmiller@rockwellcollins.com
}

Mats P. E. Heimdahl

Department of Computer Science \& Engineering, University of Minnesota

heimdahl@cs.umn.edu

\begin{abstract}
This paper describes a case study conducted to determine if formal methods could be used to validate system requirements early in the lifecycle at reasonable cost. Several hundred requirements for the mode logic of a typical Flight Guidance System were captured as natural language "shall" statements. A formal model of the mode logic was written in the RSML $^{-e}$ language and translated into the NuSMV model checker and the PVS theorem prover using translators developed as part of the project. Each "shall" statement was manually translated into a NuSMV or PVS property and proven using these tools.
\end{abstract}

\section{Introduction}

Incomplete, inaccurate, ambiguous, and volatile requirements have plagued the software industry since its inception. The avionics industry has long recognized the need for better requirements, and has spearheaded the development of several methodologies for requirements specification, including SCR [6], CoRE [4], RSML [7], and even Statecharts [5]. Despite this legacy, the requirements for most avionics systems are still specified using a combination of natural language and informal diagrams.

This paper describes a case study conducted by the Advanced Technology Center of Rockwell Collins, the Critical Systems Research Group at the University of Minnesota, and the NASA Langley Research Center to determine

This project was partially funded by the NASA Langley Research Center under contract NCC-01001 of the Aviation Safety Program 
how far formal analysis could be pushed in an industrial example [9]. In this study, a model of the mode logic of a Flight Guidance System ${ }^{1}$ was specified in the $\mathrm{RSML}^{-\boldsymbol{e}}$ notation [11]. Translators were developed from $\mathrm{RSML}^{-e}$ to the NuSMV model checker [1] and the PVS theorem prover [10, 2]. These tools were then used to verify several hundred properties of the $\mathrm{RSML}^{-e}$ model. In the process, several errors were discovered and corrected in the original RSML $^{-e}$ model.

The results of this study demonstrate that formal models can be written for real problems using notations acceptable to practicing engineers, and that formal analysis tools have matured to the point where they can be efficiently used to find errors before implementation.

\section{Requirements Modeling}

One of our first steps was to create a formal model of the black box behavior of our representative Flight Guidance System (FGS). The internal structure of a FGS can be broken down into the mode logic and the flight control laws. The flight control laws accept information about the aircraft's current and desired state and compute the pitch and roll guidance commands. The mode logic determines which lateral and vertical modes are armed and active at any given time and which flight control laws are generating guidance commands.

The formal model of the FGS was written in the $\mathrm{RSML}^{-e}$ language. When completed, it consisted of 41 input variables, 16 small, tightly synchronized hierarchical finite state machines, 122 macro or function definitions, 29 output values, and was roughly 160 pages long. A detailed description of the model and its simulation environment is available in [8].

In the course of building the $\mathrm{RSML}^{-e}$ model, we found ourselves going back and modifying the original shall statements. Sometimes, they were just wrong. More often, their organization needed to be changed to provide clear traceability to the model. Gradually, we realized that as we revised and reorganized the shall statements we produced clearer and improved description of the system. Maintaining even a coarse mapping between the shall statements and the $\mathrm{RSML}^{-e}$ model forced us to be more precise in writing down the shall statements.

\section{Model Checking}

While the FGS model could be directly translated to NuSMV using the translator developed for this project [3], the translated specification was not immediately suitable for model-checking with NuSMV due to the presence of

\footnotetext{
${ }^{1}$ While representative of the complexity of a typical system, this example did not desribed an actual fielded product.
} 
a small number of integer variables. To deal with this, we abstracted the model by hand by moving comparisons involving these variables (e.g., Altitude > PreSelectAlt + AltCapBias) into a different part of the specification and inputting the Boolean results directly into the model. Since there were only a few such computations, this took only a few hours to implement and did not significantly alter the specification. These changes reduced the state space enough that we could check almost any property of the mode logic with the NuSMV model checker in a matter of minutes.

At first, we focused on showing that our model satisfied the safety properties we had identified through a hazard analysis and fault tree analysis [12]. However, it quickly became apparent that all of the original requirements, not just the safety properties, could be stated in CTL. As a result, we extended our verification to include all the shall statements captured during elicitation.

Our approach was to state each requirement as a CTL property over the translated model. Since there was a close correspondence between names in the RSML $^{-e}$ model and the NuSMV model, this quickly became routine and most of the requirements could be translated by hand into CTL in a few minutes. All of the requirements could be specified with only two CTL formats. The first was simply a safety constraint that had to be maintained by all reachable states. The second was a constraint over a state and all possible next states. For example, the requirement If the onside FD cues are off, the onside FD cues shall be displayed when the AP is engaged was translated into the CTL property AG ( (!Onside_FD_On \& ! Is_AP_Engaged) $\rightarrow$ AX (Is_AP_Engaged -> Onside_FD_On) ).

Only these two formats were needed, largely because $\mathrm{RSML}^{-e}$ is a synchronous language in which each transition to the next system state is computed in a single atomic step. All the properties we were interested in could be stated as simple safety properties over a single state, or as a relationship describing how the system changed in a single step. If we had wanted to verify liveness properties, or if portions of the model had been allowed to evolve asynchronously, other temporal operators would have also have been needed.

Ultimately, all 281 properties originally stated informally in English were translated into CTL and checked using the NuSMV model checker. All 281 properties could be verified on a $2 \mathrm{GHz}$ Pentium 4 processor running Linux in less than an hour.

\section{Errors Found Through Model Checking}

Use of the model checker produced counter examples revealing several errors in the $\mathrm{RSML}^{-e}$ model of the mode logic that had not been discovered through simulation. One entire class of errors was discovered that involved more than one input event arriving at the same time. This could occur for a va- 
riety of reasons. For example, the pilot might press a switch at the same time the system captured a navigation source. Occasionally, these combinations would drive the model into an unsafe state.

To deal with this issue, we chose to define a prioritization on the input events so that higher priority events would supercede lower priority events. In course of developing this prioritization, we realized that it was possible for some combinations of events to be processed in the same step. For example, an input that changed the active lateral mode could often (but not always) be processed in the same step as an input that changed the active vertical mode. In other words, a partial rather than a total order of the input events was acceptable. Since we could check both the safety and functional properties of the specification with NuSMV, we felt confident that the specified behavior was correct. However, without the power of formal verification, we would never have been able to convince ourselves that the safety properties of the system were still met when such multiple input events were allowed.

\section{Theorem Proving}

We had also developed a translator to the PVS theorem prover and wanted to determine the extent of its usefulness. In contrast to model checkers, theorem provers apply rules of inference to a specification in order to derive new properties of interest. Theorem provers are generally considered harder to use than model checkers, requiring more expertise on the part of the user. However, theorem provers are not limited by the size of the state space. This makes them useful for problem domains that are not amenable to verification by model checking.

We started by using PVS to verify some of the properties already confirmed using NuSMV. In the course of completing the proofs, it became clear that we needed to define and prove many simple properties of the FGS that could be used as automatic rewrite rules by PVS. This automated and simplified the more complex proofs we were interested in. As these libraries evolved, we realized that many of these properties, as well as several useful PVS strategies (scripts defining sequences of prover commands) could have been automatically produced by the translator. These were identified as enhancements for future versions of the translator.

With this infrastructure in place, some proofs could be constructed in less than an hour. Others took several hours or even days, usually because they involved proving many other properties as intermediate lemmas. One surprise was that users proficient in PVS but unfamiliar with the FGS could usually complete a proof as quickly as someone familiar with the FGS. In fact, most of the proofs were completed by a graduate student with no avionics experience. The general process was to break the desired property down by case splits 
until a simple ASSERT or GRIND command could complete that branch of the proof tree. The structure of the proofs naturally reversed the dependency ordering defined in the $\mathrm{RSML}^{-\boldsymbol{e}}$ specification. Many of the proofs could be simplified by introducing lemmas describing how intermediate values in the dependency graph changed, but identifying such lemmas seemed to require a sound understanding the FGS mode logic. As we gained experience, we started using the dependency map produced by the $\mathrm{RSML}^{-e}$ toolset to guide us in identifying these lemmas.

Another surprise was that while the proofs might take hours to construct, they usually executed in less than twenty seconds. This was significant since the time taken to prove similar properties using the NuSMV model checker had grown steadily with the size of the model. If the model had grown much larger, it is possible that the time to verify a property using model checking might have become prohibitive. The time required to run the PVS proofs seemed much less sensitive to the size of the model.

\section{Conclusions and Future Directions}

We have described how a model of the requirements for the mode logic of a Flight Guidance System was created in the RSML $^{-e}$ language from an initial set of requirements stated as shall statements written in English. Translators were used to automatically generate equivalent models of the mode logic in the NuSMV model checker and the PVS theorem prover. The original shall statements were then hand translated into properties over these models and proven to hold over these models.

The process of creating the $\mathrm{RSML}^{-\boldsymbol{e}}$ model improved the informal requirements, and the process of proving the formal properties found errors in both the original requirements and the $\mathrm{RSML}^{-\boldsymbol{e}}$ model. The ease with which these properties were verified leads us to conclude that formal methods tools are finally maturing to the point where they can be profitably used on industrial sized problems.

Several directions exist for further work. Stronger abstraction techniques are needed to increase the classes of problems that can be verified using model checkers. Better libraries and proof strategies are needed to make theorem proving less labor intensive. More work also needs to be done to identify proof strategies and properties that can be automatically generated from the model. Since many systems consist of synchronous components connected by asynchronous buses, work needs to be done to determine how properties that span models connected by asynchronous channels can be verified. Perhaps most important, these formal verification tools need to used on real problems with commercially supported modeling tools such as SCADE, Esterel, and Simulink. 


\section{Acknowledgments}

The authors wish to acknowledge the ongoing support of this work by Ricky Butler, Kelly Hayhurst, and Celeste Bellcastro of the NASA Langley Research Center, and the efforts of Alan Tribble and Michael Whalen of Rockwell Collins and Anjali Joshi, Yunja Choi, Sanjai Rayadurgam, George Devaraj, and Dan O'Brien of the University of Minnesota for their efforts in developing this technology.

\section{References}

[1] Anonymous. NuSMV: A New Symbolic Model Checking, 2004. http://nusmv.irst.itc.it/.

[2] Anonymous. PVS home page, 2004. http://www.csl.sri.com/projects/pvs.

[3] Yunja Choi and Mats Heimdahl. Model checking RSML $^{-e}$ requirements. In Proceedings of the 7th IEEE/IEICE International Symposium on High Assurance Systems Engineering, October 2002.

[4] S. Faulk, J. Brackett, P. Ward, and J Kirby, Jr. The CoRE method for real-time requirements. IEEE Software, 9(5):22-33, September 1992.

[5] David Harel and Amnon Naamad. The STATEMATE semantics of statecharts. ACM Transactions of Software Engineering and Methodology, 5(4):293 - 333, October 1996.

[6] C.L. Heitmeyer, R.D. Jeffords, and B.G. Labaw. Automated consistency checking of requirements specifications. ACM Transactions on Software Engineering and Methodology, 5(3):231-261, July 1996.

[7] N.G. Leveson, M.P.E. Heimdahl, H. Hildreth, and J.D. Reese. Requirements Specification for Process-Control Systems. IEEE Transactions on Software Engineering, 20(9):684-706, September 1994.

[8] S. Miller, A. Tribble, T. Carlson, and E. Danielson. Flight guidance system requirements specification. Technical Report CR-2003-212426, NASA Langley Research Center, June 2003. Available at http://techreports.larc.nasa.gov/ltrs/refer/2003/cr/NASA-2003cr212426.refer.html.

[9] Steven P. Miller, Alan C. Tribble, and Mats Per Erik Heimdahl. Proving the shalls. In Keijiro Araki, Stefania Gnesi, and Dino Mandrioli, editors, Proceedigns ofthe International Symposium of Formal Methods Europe (FME 2003), volume 2805 of Lecture Notes in Computer Science, pages 75-93, Pisa, Italy, September 2003. Springer.

[10] S. Owre, J. Rushby, N. Shankar, and F. von Henke. Formal verification for fault-tolerant architectures: Prolegomena to the design of PVS. IEEE Transactions on Software Engineering, 21(2), February 1995.

[11] Jeffrey M. Thompson, Mats P.E. Heimdahl, and Steven P. Miller. Specification based prototyping for embedded systems. In Seventh ACM SIGSOFT Symposium on the Foundations on Software Engineering, number 1687 in LNCS, pages 163-179, September 1999.

[12] A. Tribble, D. Lempia, and S. Miller. Software safety analysis of a flight guidance system. In Proceedings of the 21st Digital Avionics Systems Conference (DASC'02), Irvine, CA, October 2002. 